\title{
An innovative high accuracy autonomous navigation method for the Mars rovers
}

\author{
Xujun Guan ${ }^{\mathrm{a}}$, Xinlong Wang ${ }^{\mathrm{a}, *}$, Jiancheng Fang ${ }^{\mathrm{b}}$, Shaojun Feng ${ }^{\mathrm{c}}$
}

\author{
a School of Astronautics, Beihang University, Beijing 100191, China \\ ${ }^{b}$ School of Instrumentation Science \& Opto-electronics Engineering, Beihang University, Beijing 100191, China \\ ${ }^{c}$ Centre for Transport Studies, Department of Civil and Environmental Engineering, Imperial College London, SW7 2AZ, United Kingdom
}

\author{
A b s t r a c t
}

Autonomous navigation is an important function for a Mars rover to fulfill missions successfully. It is a critical technique to overcome the limitations of ground tracking and control traditionally used. This paper proposes an innovative method based on SINS (Strapdown Inertial Navigation System) with the aid of star sensors to accurately determine the rover's position and attitude. This method consists of two parts: the initial alignment and navigation. The alignment consists of a coarse position and attitude initial alignment approach and fine initial alignment approach. The coarse one is used to determine approximate position and attitude for the rover. This is followed by fine alignment to tune the approximate solution to accurate one. Upon the completion of initial alignment, the system can be used to provide real-time navigation solutions for the rover. An autonomous navigation algorithm is proposed to estimate and compensate the accumulated errors of SINS in real time. High accuracy attitude information from star sensor is used to correct errors in SINS. Simulation results demonstrate that the proposed methods can achieve a high precision autonomous navigation for Mars rovers.

\section{Introduction}

The Mars rover is an extremely important tool for the Mars exploration [1]. Some scientific missions, such as the collection of the environment materials, rock and soil samples, can be accomplished by the rover's exploration [2]. Usually, the rover is controlled by radio signals from the stations on the Earth. However, the control signals may be blocked by the two planets (Earth and Mars) due to the relative motion (including rotation) of the two. Consequently, the rover cannot be controlled all the time by earth stations [3]. Even during the period the control signals can reach the rover, there is still delay in the communication resulting latency in the response of rover. Therefore, it is very crucial to develop autonomous for Mars rover including autonomous navigation. The rover's autonomous navigation includes two steps, the initial alignment and navigation.

The rover's initial position and attitude provide a basic reference. The errors in initial alignment have a direct impact on the rover's navigation solutions. Currently, there are a few methods on the determination of rover's initial position including the radio ranging technique $[4,5]$ and landmark based autonomous position and attitude determination method [6]. The radio ranging technique has the advantage of global absolute positioning, however it cannot perform autonomous positioning in real time. For example the Spirit and Opportunity rovers used their radio system to communicate directly with earth stations after land on the Mars. The rovers' locations were determined by two-way Doppler signals obtained by earth stations and 
the orbiter of Odyssey from the second Martian day to the fourth Martian day. The landmark based autonomous position and attitude determination method estimates the state of the rover by referring crater as navigation landmark. The Spirit and Opportunity rovers' horizontal velocities with respect to the surface of Mars are estimated by matching the landmarks between two neighboring images during landing. However, the performance of this method depends on positioning accuracy of the landmarks.

The main techniques for the rover's autonomous navigation include inertial navigation [7-9], vision navigation $[10,11]$ and celestial navigation [12-17]. The inertial navigation has the advantages of immune from environment effects, high precision in short time and real-time autonomy. However, the errors in navigation solutions accumulate over time. One example is that the Spirit and Opportunity rovers employ Litton LN-200, an inertial Measurement Unit (IMU) [18]. The gyros and accelerometers in the IMU are used to determine the attitude. The wheel-odometry together with IMU is used to derive the position. Vision navigation tracks the motion of autonomously-selected "interesting" terrain features between two pairs of stereo images in both 2dimensional pixel coordinates and 3-dimensional coordinates. Spirit and Opportunity rovers use visual odometry system which can achieve navigation solutions in real-time. It has the advantages of strong autonomy and high precision, which can be used to correct the position and attitude errors due to the IMU errors and the wheel-slippage. However, this method incurs large amount of calculation. It can only be used in a local area. Furthermore, the performance of this method depends largely on terrain features. Celestial navigation system (CNS) can provide position and attitude solutions without accumulated errors. However, it is restricted by the visibility of referencing stars. Furthermore, its data update rate is relatively low. The Spirit and Opportunity rovers use the Panoramic Camera as a vision sensor to gain the image of the sun. The rover's true north can be calculated by determining the centroid position of the sun in the image.

Apparently, none of the above navigation techniques can meet therequirements of the rover'sautonomousnavigation due to their weaknesses. Consequently, a number of inte-grated techniques have been developed. Among them, SINS/CNS integrated navigation approach has been extensively investigated. In [19], an autonomous navigation approach for small satellites was introduced based on information fusion of starlight sensor, geomagnetic meter and gyros. This approach improves the accuracy of attitude determination for small satellites by using the accurate starlight vectors to correct the errors in gyros measurements. In [20], a high precision and reliable mathematical horizon reference was proposed. Based on this reference, a SINS/CNS deep integrated navigation for cruise missile is proposed to achieve higher navigation accuracy. An ensemble neural network based SINS/CNS integrated navigation system for ballistic missile was shown in [21]. I n [22], two different algorithms were presented for the estimation of spacecraft body angular rates in the absence of gyro rate data with a star tracker. Another attitude determina-tion method for spacecraft with star trackers was provided in [23]. This method gives the relationship between the obtain-able accuracy and the numbers of stars. In [24], a s u n s e n s o r for planetary rover absolute heading detection was presented. It solves the problem of noisy rotational-speed measurements integrated with time. In [25], a two-mode INS/CNS navigation method for Lunar Rovers was provided. When the rover is stationary, a tightly coupled mode is used to correct the position and attitude of the rover. When the rover is in motion, a loosely coupled mode is used to correct the attitude of the rover. In all of the methods above, the celestial navigation method is always used to correct the errors of gyros and accelerometers, and determine the vehicle's attitude and position. However, the initial alignment of SINS with the assistance of CNS has not been studied intensively. In addition, the fusion of information from SINS and CNS to achieve a long time stable, high accuracy and reliable autonomous navigation for rovers is still a challenge.

This paper proposes a star sensor based initial alignment method for SINS. A SINS and star sensor based autonomous navigation method for the rover is proposed to achieve high accuracy navigation solutions.

\section{Initial alignment of a rover}

The initial alignment is to determine the initial position and attitude of a rover before its navigation system can perform the navigation function. The initial position is the point where the position solutions refer to during the navigation process. The initial attitude is the reference of rotation from which the relative rotations can be taken into account for the position calculation. The initial alignment of SINS can be achieved in static or dynamic mode. In dynamic mode, the rover's position and attitude can be informed or determined by other systems. In the static mode, the initial alignment can be achieved with sensors onboard. Since the position of the rover is not change and the velocity is zero, together with other information, the output of the SINS can be used to estimate the inertial sensor errors.

\subsection{The definition of initial position and attitude}

There are a number of coordinate systems in navigation on Mars. The relevant coordinate systems are introduced as follows:

Martian inertial coordinate system $i$ has its origin at the center of mass of Mars. Its orientation is that of the International Celestial Reference System.

Martian fixed coordinate system $m$ has its origin at the center of mass of Mars. Its $X$-axis is in the direction of the intersection of the Prime Meridian and it lies in the equatorial plane, $Z$-axis is in the direction of the north pole, and $Y$-axis forms a right-hand system with the other axes.

Rover body coordinate system $b$ has its origin at the center of the rover. The $X Y$ plane is parallel to the plane of the rover deck. The $Z$-axis points up, the $Y$-axis points forward, and the $X$-axis is determined using the right-hand rule. This frame moves with the rover.

Navigation coordinate system $n$ has its origin coincided with the Rover body coordinate system. The $X$-axis points east, the $Y$-axis points north, and the $Z$-axis is in the opposite direction of gravity. 
Horizontal coordinate system $h$ is locally level, and the coordinate system $n$ rotates through the yaw angle about the local vertical is the coordinate system $h$.

For coordinate can be transformed from one to another by a direction cosine matrix (DCM). For example $\mathbf{C}_{a}^{d}$ represent a transformation from the coordinate system $a$ to the coordinate system $d$.

The rover's initial position refers to the latitude and longitude of its starting point (the landing point of the rover) in the coordinate system $m$. The relationship between the position and the DCM $\mathbf{C}_{m}^{n}$ is shown in Eq. (1). Similarly, the rover's initial attitude can be expressed as the DCM $\mathbf{C}_{n}^{b}$. It is shown in Eq. (2).

$\boldsymbol{C}_{m}^{n}=\left[\begin{array}{ccc}-\sin \lambda & \cos \lambda & 0 \\ -\cos \lambda \sin L & -\sin \lambda \sin L & \cos L \\ \cos \lambda \cos L & \sin \lambda \cos L & \sin L\end{array}\right]$
$\boldsymbol{C}_{n}^{b}=\left[\begin{array}{ccc}c \gamma c \psi+s \gamma s \theta s \psi & -c \gamma s \psi+s \gamma s \theta c \psi & -s \gamma c \theta \\ c \theta s \psi & c \theta c \psi & s \theta \\ s \gamma c \psi-c \gamma s \theta s \psi & -s \gamma s \psi-c \gamma s \theta c \psi & c \gamma c \theta\end{array}\right]$

where $\lambda, L$ represent the rover's latitude and longitude, respectively, $\theta, \gamma, \psi$ represent the rover's pitch angle, roll angle and yaw angle, respectively, $c \theta$ and $s \theta$ represent, respectively, the cosine and sine of $\theta$, others and so on.

\subsection{Coarse alignment method}

2.2.1. Determination of the rover's pitch angle and roll angle

The rover is stationary in the process of initial alignment. According to the accelerometers readings $\left[\begin{array}{lll}f_{x}^{b} & f_{y}^{b} & f_{z}^{b}\end{array}\right]^{T}$, we can indirectly obtain the local gravity vector in the coordinate system $b$. And the local gravity vector in the coordinate system $n$ is $\left[\begin{array}{lll}0 & 0 & -g\end{array}\right]^{T}$. The relationship between them is shown as follows:

$\left[\begin{array}{l}f_{x}^{b} \\ f_{y}^{b} \\ f_{z}^{b}\end{array}\right]=\boldsymbol{C}_{n}^{b}\left[\begin{array}{l}0 \\ 0 \\ g\end{array}\right]=\left[\begin{array}{c}-\cos \theta \sin \gamma \\ \sin \theta \\ \cos \theta \cos \gamma\end{array}\right] g$

In application, we constrain $\theta$ to the range $-\pi / 2 \leq$ $\theta \leq \pi / 2, \gamma$ to the range $-\pi \leq \gamma \leq \pi$. And according to Eq. (3), we can obtain that:

$\theta=\arcsin \left(f_{y}^{b} / g\right)$

$\gamma=a \tan 2\left(-f_{x}^{b}, f_{z}^{b}\right)$

\subsubsection{Determination of the rover's initial position and yaw angle}

It is very easy to simultaneously observe three or more guide stars with new large field of view star sensor. Without the need of any external reference information, it can directly provide precise attitude information of the vehicle with respect to the inertial space, and the precision is stable in the whole process. Therefore, according to the sensor's output, the precise DCM $\widetilde{\mathbf{C}}_{i}^{b}$ can be obtained. According to the current time, we can obtain the DCM $\mathbf{C}_{i}^{m}$. The DCM $\mathbf{C}_{h}^{b}$ can be determined by the use of the known pitch angle and roll angle. In a word, we can obtain:

$\widetilde{\boldsymbol{C}}_{i}^{b}=\boldsymbol{C}_{h}^{b} \boldsymbol{C}_{m}^{h} \boldsymbol{C}_{i}^{m}$

Therefore, $\boldsymbol{C}_{m}^{h}=\left(\boldsymbol{C}_{h}^{b}\right)^{T} \widetilde{\boldsymbol{C}}_{i}^{b}\left(\boldsymbol{C}_{i}^{m}\right)^{T}$.

The transformation from the coordinate system $m$ to the coordinate system $h$ can be carried out as three successive rotations about different axes. First, the coordinate system $m$ rotates through $\left(90^{\circ}+\lambda\right)$ degree about its $Z_{m}$ axis to the $O X_{1} Y_{1} Z_{1}$ coordinate system. And then the $O X_{1} Y_{1} Z_{1}$ coordinate system rotates through $\left(90^{\circ}-L\right)$ degree about $X_{1}$ axis to the coordinate system $n$. Finally, the coordinate system $n$ rotates through $-\psi$ degree about its $Z_{n}$ axis to the coordinate system $h$. So, the relationship between the matrix $\boldsymbol{C}_{m}^{h}$ and the rover's latitude $L$, longitude $\lambda$ and yaw angle $\psi$ is shown as follows:

$\boldsymbol{C}_{m}^{h}=\left[\begin{array}{lll}C_{11} & C_{12} & C_{13} \\ C_{21} & C_{22} & C_{23} \\ C_{31} & C_{32} & C_{33}\end{array}\right]=\left[\begin{array}{ccc}c \lambda s L s \psi-s \lambda C \psi & c \lambda c \psi+s \lambda s L S \psi & -s \psi c L \\ -c \lambda s L c \psi-s \lambda s \psi & c \lambda s \psi-s \lambda s L c \psi & c \psi c L \\ c \lambda c L & s \lambda c L & s L\end{array}\right]$

where $c \lambda$ and $s \lambda$ represent, respectively, the cosine and sine of $\lambda$, others and so on.

Without loss of generality, we constrain $L$ to the range $-\pi / 2 \leq L \leq \pi / 2, \lambda$ to the range $-\pi \leq \lambda \leq \pi$, and $\psi$ to the range $0 \leq \psi \leq 2 \pi$. So according to Eq. (7), the following equations can be obtained:

$\lambda=a \tan 2\left(C_{32}, C_{31}\right)$

$L=\operatorname{arc} \sin \left(C_{33}\right)$

$\psi= \begin{cases}a \tan 2\left(-C_{13}, C_{23}\right) & C_{13}<0 \\ a \tan 2\left(-C_{13}, C_{23}\right)+2 \pi & C_{13}>0\end{cases}$

When the constant bias and random bias of each accelerometer are chosen as $10 \mu \mathrm{g}$ and $5 \mu \mathrm{g}$, respectively, and the measurement noise of star sensor is $2 "$. The errors of rover's position and attitude determined by the coarse initial alignment approach at $30^{\circ} \mathrm{N}$ latitude and $110^{\circ} \mathrm{E}$ longitude are shown in Table 1.

\subsection{Fine alignment method}

From the analysis above, the coarse alignment method is very easy and can obtain the rover's initial position and attitude in a short time. But it cannot eliminate the influence of measurement noise, nor can it calibrate the gyro drift.

Hence, on the basis of coarse alignment method, a fine one is proposed. The SINS linear error model is used as system state equation, and the velocity errors outputted by SINS are used as observation variables. In addition, the DCM $\widetilde{\boldsymbol{C}}_{i}^{b}$ determined by star sensor is fused with the DCM $\hat{\boldsymbol{C}}_{i}^{b}$ determined by SINS. All of these make it possible that platform misalignment angle, latitude error and longitude

Table 1

Accuracy of the coarse position and attitude initial alignment approach

\begin{tabular}{llllll}
\hline Pose & Longitude & Latitude & Pitch angle & Roll angle & Yaw angle \\
\hline Error(") & 3.5016 & -1.1672 & 2.5175 & -2.3633 & 2.2837 \\
\hline
\end{tabular}




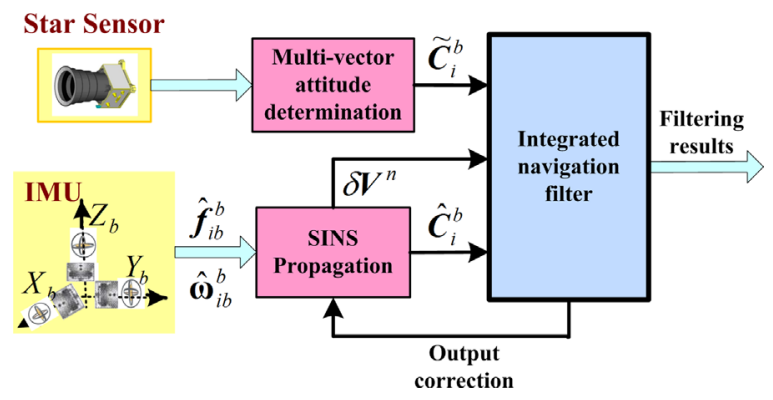

Fig. 1. The working principle of fine position and attitude initial alignment approach.

error can be estimated well. And these estimates are subsequently used to correct the rover's initial attitude and position errors. Moreover, this method can obtain the estimates of gyros drift. The working principle of this method is shown in Fig. 1:

\subsubsection{Attitude error model}

When choose east-north-up geographical coordinate system as the navigation coordinate system, the attitude error model can be expressed as follows:

$\dot{\boldsymbol{\varphi}}^{n}=\delta \boldsymbol{\omega}_{m n}^{n}-\left(\boldsymbol{\omega}_{i m}^{n}+\boldsymbol{\omega}_{m n}^{n}\right) \times \boldsymbol{\varphi}^{n}-\boldsymbol{C}_{b}^{n} \delta \boldsymbol{\omega}_{i b}^{b}$

where $\boldsymbol{\varphi}^{n}$ is the platform misalignment angle vector, $\delta \boldsymbol{\omega}_{i b}^{b}$ is the measurement error vector of gyros.

We employ Farrenkopf's gyro dynamics error model
2.3.2. Velocity and position error model

Velocity error equation is given as follows:

$$
\begin{aligned}
\delta \dot{\boldsymbol{V}}^{n}= & \boldsymbol{f}^{n} \times \boldsymbol{\varphi}^{n}+\boldsymbol{C}_{b}^{n} \nabla^{b}-\left(2 \boldsymbol{\omega}_{i m}^{n}+\boldsymbol{\omega}_{m n}^{n}\right) \\
& \times \delta \boldsymbol{V}^{n}-\left(2 \delta \boldsymbol{\omega}_{i m}^{n}+\delta \boldsymbol{\omega}_{m n}^{n}\right) \times \boldsymbol{V}^{n}
\end{aligned}
$$

where $\delta \boldsymbol{V}^{n}$ is the velocity error vector, $\nabla^{b}$ is the accelerometer constant bias vector.

Position error equation is given as follows:

$\left\{\begin{array}{l}\delta \dot{\lambda}=\delta v_{E} \sec \quad L / R_{m}+v_{E} \sec \quad L \text { tan } L \delta L / R_{m} \\ \delta \dot{L}=\delta v_{N} / R_{m}\end{array}\right.$

where $\delta \lambda$ and $\delta L$ are the latitude error and longitude error, respectively. $R_{m}$ is the radius of Mars.

\subsubsection{System error state equation}

According to the attitude error model, gyro error model, velocity error equation and position error Eqs. (11)-(14), the system state equation can be established as follows:

$\dot{\boldsymbol{X}}(t)=\boldsymbol{F}(t) \boldsymbol{X}(t)+\boldsymbol{G}(t) \boldsymbol{W}(t)$

where the system state vector is:

$\boldsymbol{X}=\left[\begin{array}{llllllllllllll}\phi_{E} & \phi_{N} & \phi_{U} & \delta v_{E} & \delta v_{N} & \delta v_{U} & \delta \lambda & \delta L & \varepsilon_{x} & \varepsilon_{y} & \varepsilon_{z} & \nabla_{x} & \nabla_{y} & \nabla_{z}\end{array}\right]^{T}$,

where the system noise vector is:

$\boldsymbol{W}(t)=\left[\begin{array}{lllllllll}w_{g x} & w_{g y} & w_{g z} & w_{a x} & w_{a y} & w_{a z} & w_{\varepsilon x} & w_{\varepsilon y} & w_{\varepsilon z}\end{array}\right]^{T}$

$\boldsymbol{F}(t)$ is system state matrix, $\boldsymbol{G}(t)$ is system noise drive matrix. $\boldsymbol{F}(t)$ and $\boldsymbol{G}(t)$ can be, respectively expressed as follows:

$$
\begin{aligned}
& \boldsymbol{F}(t)=\left[\begin{array}{cc}
\boldsymbol{F}_{N} & \boldsymbol{F}_{S} \\
\mathbf{0}_{6 \times 8} & \mathbf{0}_{6 \times 6}
\end{array}\right]_{14 \times 14} \boldsymbol{F}_{S}=\left[\begin{array}{cc}
-\boldsymbol{C}_{b}^{n} & \mathbf{0}_{3 \times 3} \\
\mathbf{0}_{3 \times 3} & \boldsymbol{C}_{b}^{n} \\
\mathbf{0}_{2 \times 3} & \mathbf{0}_{2 \times 3}
\end{array}\right]
\end{aligned}
$$

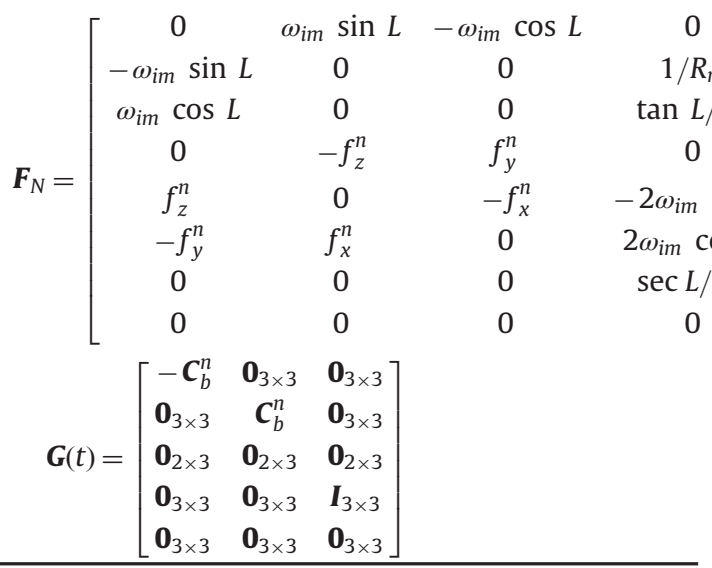

$\left.\begin{array}{cccc}1 / R_{m} & 0 & 0 & 0 \\ 0 & 0 & 0 & -\omega_{\text {im }} \sin L \\ 0 & 0 & 0 & \omega_{i m} \cos L \\ 2 \omega_{\text {im }} \sin L & -2 \omega_{\text {im }} \cos L & 0 & 0 \\ 0 & 0 & 0 & 0 \\ 0 & 0 & 0 & 0 \\ 0 & 0 & 0 & 0 \\ 1 / R_{m} & 0 & 0 & 0\end{array}\right]$

[26], which means that we ignore the output noise for rate-integrating gyros. This is an excellent approximation for navigation-grade gyros [27].

$\left\{\begin{array}{l}\boldsymbol{\omega}_{i b(\text { gyro })}^{b}=\omega_{i b(\text { true })}^{b}+\varepsilon+\eta_{1} \\ \dot{\varepsilon}=\eta_{2}\end{array}\right.$

where $\omega_{i b(\text { gyro })}^{b}$ is the gyro output vector, $\boldsymbol{\omega}_{i b(t r u e)}^{b}$ is the true angular rate vector, $\varepsilon$ is the gyro drift vector, and $\eta_{1}$ and $\eta_{2}$ are zero-mean white noise processes.

\subsubsection{System measurement equation}

Quaternion is selected to express the attitude, and the attitude kinematics model is:

$$
\left\{\begin{array}{l}
\dot{\boldsymbol{Q}}=\frac{1}{2} \boldsymbol{Q} \otimes \boldsymbol{\omega} \\
\boldsymbol{Q}=\left[\begin{array}{l}
\boldsymbol{q} \\
q_{4}
\end{array}\right]
\end{array}\right.
$$


The quaternion components obey the unit length constraint:

$$
|\boldsymbol{Q}|^{2}=|\boldsymbol{q}|^{2}+q_{4}^{2}=1
$$

In order to relax the quaternion normalization requirement of Eq. (17), we parameterize the attitude matrix $\hat{\boldsymbol{C}}_{n}^{b}$ by:

$\hat{\boldsymbol{C}}_{n}^{b}=|\boldsymbol{Q}|^{-2}\left\{\left(q_{4}^{2}-|\boldsymbol{q}|^{2}\right) \boldsymbol{I}_{3 \times 3}-2 q_{4}[\mathbf{q} \times]+2 \mathbf{q} \boldsymbol{q}^{T}\right\}$

where $\boldsymbol{I}_{3 \times 3}$ is the $3 \times 3$ identity matrix and the cross product matrix is:

$[\boldsymbol{q} \times]=\left[\begin{array}{ccc}0 & -q_{3} & q_{2} \\ q_{3} & 0 & -q_{1} \\ -q_{2} & q_{1} & 0\end{array}\right]$

The matrix $\boldsymbol{C}_{i}^{m}$ combined with the strapdown matrix $\hat{\boldsymbol{C}}_{n}^{b}$ and the position matrix $\hat{\boldsymbol{C}}_{m}^{n}$, we can get that:

$\hat{\boldsymbol{C}}_{i}^{b}=\hat{\boldsymbol{C}}_{n}^{b} \hat{\boldsymbol{C}}_{m}^{n} \boldsymbol{C}_{i}^{m}$

Due to the influence of alignment errors and gyros drift, there is a misalignment angle vector $\varphi^{n}=\left[\begin{array}{lll}\varphi_{E} & \varphi_{N} & \varphi_{U}\end{array}\right]^{T}$ between the platform coordinate system and the coordinate system $n$. The following equation can be obtained:

$\boldsymbol{C}_{n}^{b}=\hat{\boldsymbol{C}}_{n}^{b}\left(\boldsymbol{I}_{3 \times 3}-\left[\boldsymbol{\varphi}^{n} \times\right]\right)$

Because of the position errors $\delta L, \delta \lambda$, the computed geographical coordinate system does not coincide with the normal geographical coordinate system, and there is a position error vector $\delta \mathbf{P}=\left[\begin{array}{llll}-\delta L & \delta \lambda \cdot \cos L & \delta \lambda \cdot \sin L\end{array}\right]^{T}$ between them. The following equation can be obtained:

$\boldsymbol{C}_{m}^{n}=\left(\boldsymbol{I}_{3 \times 3}+[\delta \boldsymbol{P} \times]\right) \hat{\boldsymbol{C}}_{m}^{n}$

According to Eqs. (20) and (21), the errors of DCM $\hat{\boldsymbol{C}}_{i}^{b}$ determined by SINS is mainly caused by the platform misalignment angle vector $\varphi^{n}$ and the position errors $\delta L, \delta \lambda$.

We treat the best-fit quaternion estimate of $\widetilde{\boldsymbol{C}}_{i}^{b}$ as the composition of the true rotation $\mathbf{C}_{i}^{b}$ and a measurement error $\delta \mathbf{C}_{i}^{b}$ :

$$
\left\{\begin{array}{l}
\widetilde{\mathbf{C}}_{i}^{b}=\delta \mathbf{C}_{i}^{b} \mathbf{C}_{i}^{b} \\
\delta \mathbf{C}_{i}^{b}=\mathbf{I}_{3 \times 3}-[\delta \boldsymbol{\theta} \times]
\end{array}\right.
$$

where,

$[\delta \boldsymbol{\theta} \times]=\left[\begin{array}{ccc}0 & -\delta \theta_{z} & \delta \theta_{y} \\ \delta \theta_{z} & 0 & -\delta \theta_{x} \\ -\delta \theta_{y} & \delta \theta_{x} & 0\end{array}\right]$

The product of the matrix $\hat{\mathbf{C}}_{i}^{b}$ and the matrix $\widetilde{\boldsymbol{C}}_{i}^{b}$ is denoted as measurement variable $\mathbf{Z}_{s}$, so according to Eqs. (19)-(22), we can get that:

$$
\begin{aligned}
\boldsymbol{Z}_{s} & =\hat{\boldsymbol{C}}_{i}^{b} *\left(\widetilde{\boldsymbol{C}}_{i}^{b}\right)^{T}=\hat{\boldsymbol{C}}_{i}^{b} *\left(\left(\boldsymbol{I}_{3 \times 3}-[\delta \boldsymbol{\theta} \times]\right) \boldsymbol{C}_{i}^{b}\right)^{T}=\hat{\boldsymbol{C}}_{i}^{b} *\left(\boldsymbol{C}_{i}^{b}\right)^{T}+[\delta \boldsymbol{\theta} \times] \\
& =\left(\hat{\boldsymbol{C}}_{n}^{b} \hat{\boldsymbol{C}}_{m}^{n} \boldsymbol{C}_{i}^{m}\right) *\left(\hat{\boldsymbol{C}}_{n}^{b}\left(\boldsymbol{I}_{3 \times 3}-\left[\boldsymbol{\varphi}^{n} \times\right]\right)\left(\boldsymbol{I}_{3 \times 3}+[\delta \boldsymbol{P} \times]\right) \hat{\boldsymbol{C}}_{m}^{n} \boldsymbol{C}_{i}^{m}\right)^{T}+[\delta \boldsymbol{\theta} \times] \\
& =\boldsymbol{I}_{3 \times 3}+\hat{\boldsymbol{C}}_{n}^{b}\left[\boldsymbol{\varphi}^{n} \times\right]\left(\hat{\boldsymbol{C}}_{n}^{b}\right)^{T}-\hat{\boldsymbol{C}}_{n}^{b}[\delta \boldsymbol{P} \times]\left(\hat{\boldsymbol{C}}_{n}^{b}\right)^{T}+[\delta \boldsymbol{\theta} \times]
\end{aligned}
$$

where $\boldsymbol{Z}_{s}$ is a anti-symmetric matrix, which can be described as follows:

$\boldsymbol{Z}_{s}=\left[\begin{array}{ccc}1 & -Z_{z} & Z_{y} \\ Z_{z} & 1 & -Z_{x} \\ -Z_{y} & Z_{x} & 1\end{array}\right]$

We utilize $Z_{1}=\left[\begin{array}{lll}Z_{x} & Z_{y} & Z_{z}\end{array}\right]^{T}$ as measurement, and in combination with the system state vector $\boldsymbol{X}$, the system measurement equation can be expressed as follows:

$\boldsymbol{Z}_{1}=\left[\begin{array}{l}0.5 *\left[Z_{s}(3,2)-Z_{s}(2,3)\right] \\ 0.5 *\left[Z_{s}(1,3)-Z_{s}(3,1)\right] \\ 0.5 *\left[Z_{s}(2,1)-Z_{s}(1,2)\right]\end{array}\right]=\boldsymbol{H}_{1} \boldsymbol{X}+\boldsymbol{V}_{1}$

where, $\boldsymbol{H}_{1}=\left[\begin{array}{llll}\boldsymbol{H}_{11} & \mathbf{0}_{3 \times 3} & \boldsymbol{H}_{13} & \mathbf{0}_{3 \times 6}\end{array}\right]$ is the attitude measurement matrix, $\boldsymbol{V}_{1}$ is the white noise vector.

Let $\hat{\boldsymbol{C}}_{n}^{b}=\left[\begin{array}{lll}T_{11} & T_{12} & T_{13} \\ T_{21} & T_{22} & T_{23} \\ T_{31} & T_{32} & T_{33}\end{array}\right]$, and according to Eq. (23):

$\boldsymbol{H}_{11}=\left[\begin{array}{lll}T_{22} T_{33}-T_{23} T_{32} & T_{23} T_{31}-T_{21} T_{33} & T_{21} T_{32}-T_{22} T_{31} \\ T_{13} T_{32}-T_{12} T_{33} & T_{11} T_{33}-T_{13} T_{31} & T_{12} T_{31}-T_{11} T_{32} \\ T_{12} T_{23}-T_{13} T_{22} & T_{13} T_{21}-T_{11} T_{23} & T_{11} T_{22}-T_{12} T_{21}\end{array}\right]$,

$\boldsymbol{H}_{13}=\boldsymbol{H}_{11} \times\left[\begin{array}{cc}0 & 1 \\ -\cos L & 0 \\ -\sin L & 0\end{array}\right]$

The velocity errors outputted by SINS are used as the velocity error measurement $\boldsymbol{Z}_{2}$. Combined with the system state vector $\boldsymbol{X}$, another measurement equation can be expressed as follows:

$\boldsymbol{Z}_{2}=\left[\begin{array}{l}\delta v_{E} \\ \delta v_{N} \\ \delta v_{U}\end{array}\right]=\boldsymbol{H}_{2} \boldsymbol{X}+\boldsymbol{V}_{2}$

where $\boldsymbol{H}_{2}=\left[\begin{array}{llll}\mathbf{0}_{3 \times 3} & \boldsymbol{I}_{3 \times 3} & \mathbf{0}_{3 \times 2} & \mathbf{0}_{3 \times 6}\end{array}\right]$ is the velocity error measurement matrix, and $\mathbf{V}_{2}$ is the measurement noise.

According to the measurement equations (24) and (25), the system measurement equation can be established as follows:

$\boldsymbol{Z}=\left[\begin{array}{l}\boldsymbol{Z}_{1} \\ \boldsymbol{Z}_{2}\end{array}\right]=\boldsymbol{H} \boldsymbol{X}+\boldsymbol{V}$

where, $\boldsymbol{H}=\left[\begin{array}{llll}\boldsymbol{H}_{11} & \mathbf{0}_{3 \times 3} & \boldsymbol{H}_{13} & \mathbf{0}_{3 \times 6} \\ \mathbf{0}_{3 \times 3} & \boldsymbol{I}_{3 \times 3} & \mathbf{0}_{3 \times 2} & \mathbf{0}_{3 \times 6}\end{array}\right], \boldsymbol{V}$ is the measurement noise.

\subsubsection{Simulation verification and analysis}

The initial position and attitude of simulation are the results of coarse alignment method. The errors of IMU are: the constant bias and random bias of each accelerometer are chosen as $10 \mu \mathrm{g}$ and $5 \mu \mathrm{g}$, respectively. The standard deviation of each gyro measurement noise are $0.005^{\circ} / \mathrm{h}$, the angular random walk of each gyro are $0.01^{\circ} \mathrm{h}$, with a driving noise whose standard deviation is $0.001^{\circ} / \mathrm{h}$. The update rates of accelerometers and gyros are $100 \mathrm{~Hz}$ 


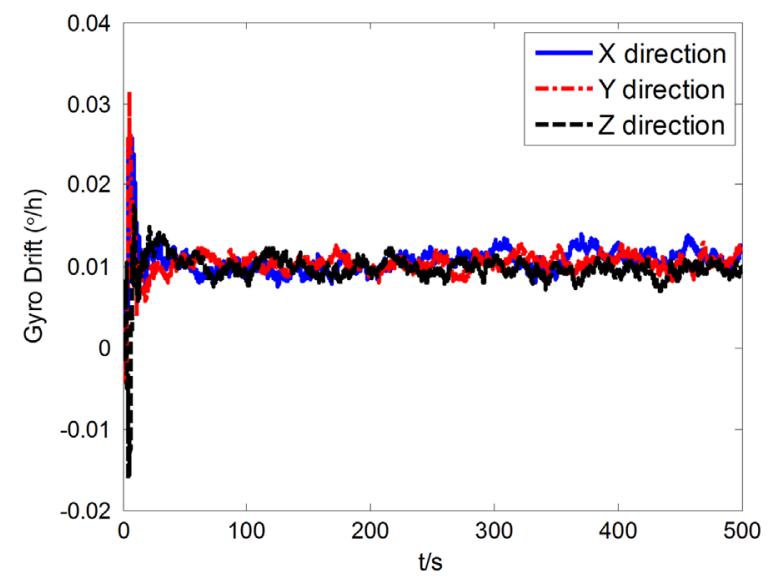

Fig. 2. The estimates of gyro drift.

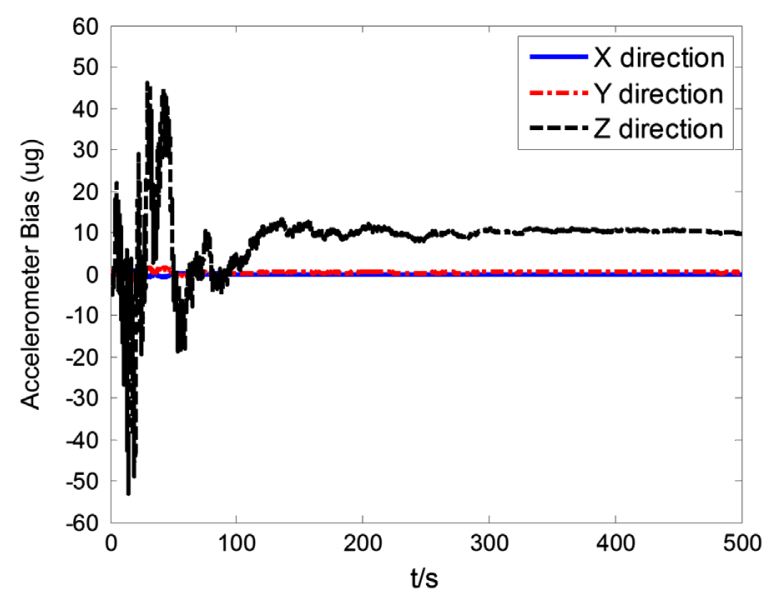

Fig. 3. The estimates of accelerometer constant bias.

The star sensor can output the rover's inertial attitude with the accuracy of $2 "$, and its update rate is $1 \mathrm{~Hz}$. The simulation results are shown as follows:

According to Eq. (11), we can see that there is a coupling relationship between the misalignment angle and the gyro drift. Because the accuracy of matrix $\widetilde{\boldsymbol{C}}_{i}^{b}$ determined by star sensor is very high, misalignment angle can be estimated in high accuracy by fusing the matrix $\widetilde{\boldsymbol{C}}_{i}^{b}$ with the matrix $\hat{\boldsymbol{C}}_{i}^{b}$. Therefore, $\boldsymbol{\varepsilon}^{b}$ can be estimated well too. As can be seen from Fig. 2, the estimate of $\boldsymbol{\varepsilon}^{b}$ extremely approximates to the normal one. The simulation results coincide with the theory. According to Eq. (13), we can see that $\nabla_{x}^{n}-g \phi_{y}=0$ and $\nabla_{y}^{n}+g \phi_{x}=0$ can be got when $\delta \dot{\boldsymbol{V}}^{n}=0$. Therefore, $\nabla_{x}^{n}$ and $\nabla_{y}^{n}$ can't be estimated. As can be seen from Fig. 3, only $\nabla_{z}^{b}$ can be estimated well, the other two can't be estimated. This is mainly resulted from $\nabla_{x}^{n}$ and $\nabla_{y}^{n}$ can't be estimated, which coincide with the theory. The estimated errors of $\phi_{x}$ and $\phi_{y}$ are approximately $2^{\prime \prime}$ when $\nabla_{x}^{n}$ and $\nabla_{y}^{n}$ can't be estimated (because of the constant bias of each accelerometer is $10 \mu \mathrm{g}$ ). And this finally leads to the errors of pitch angle and roll angle are about 2", as shown in Fig. 4. By using the estimates of $\delta L$ and $\delta \lambda$ to correct the latitude and longitude information, respectively, Fig. 5 can show: the longitude error is about

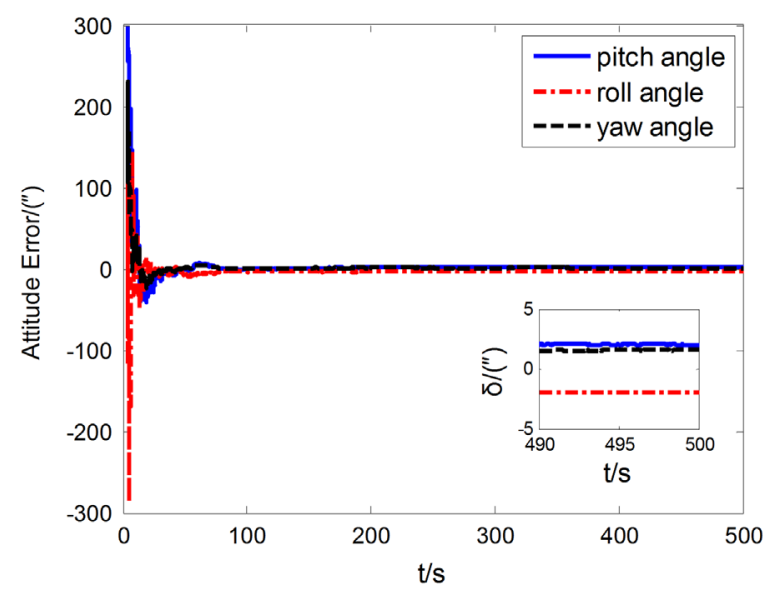

Fig. 4. The attitude errors.

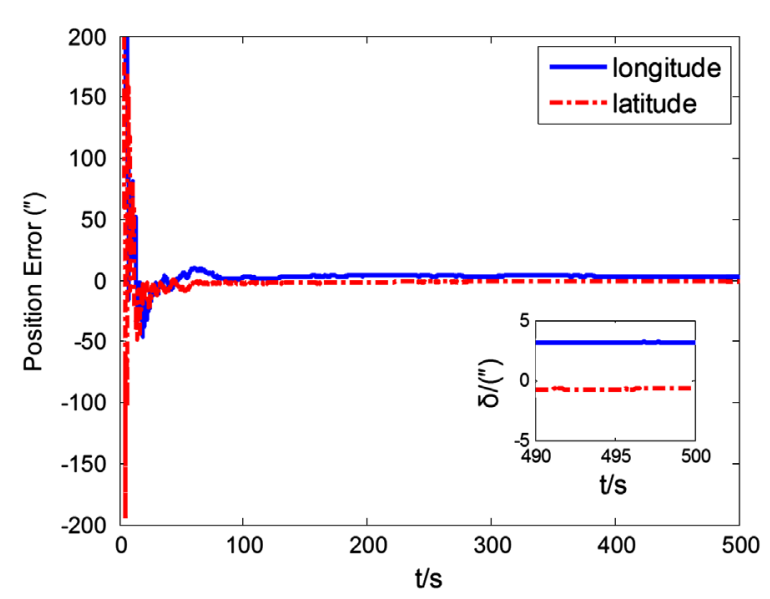

Fig. 5. The latitude error and longitude error.

$3 "$, and the latitude error is less than $1^{\prime \prime}$. Compared with the coarse alignment method, the positioning accuracy of the fine one is improved. Moreover, it can also obtain the estimates of $\varepsilon^{b}$.

\section{The navigation solutions}

From the analysis above, the presented initial alignment method based on SINS with the aid of star sensors can initialize the rover's position and attitude with high accuracy. In this method, a coarse initial alignment approach is first used to determine approximate position and attitude for the rover. A fine alignment approach is followed to tune the approximate solution to accurate one.

In addition, star sensor is a highly precise attitude sensor by observing stars, and it can provide arc-second level and even more precise attitude information. When the rover is in motion, an autonomous navigation method is used to estimate and compensate the SINS errors in real time. In this method, the matrices $\widetilde{\boldsymbol{C}}_{i}^{b}$ and $\hat{\boldsymbol{C}}_{i}^{b}$ are fused to take the advantages of both inertial navigation and celestial navigation. And the transverse and vertical velocities in rover body coordinate system are zero in theory, which is used as limiting condition to 


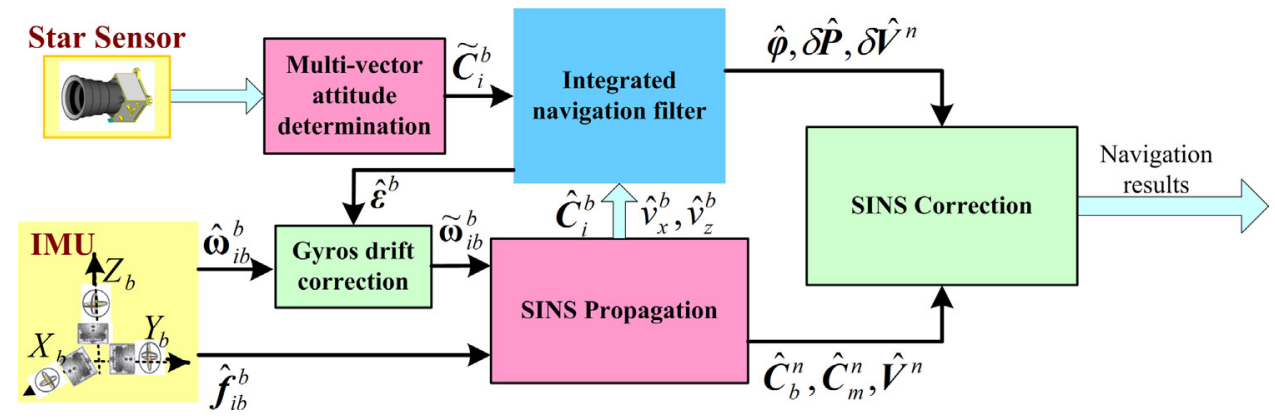

Fig. 6. The working principle of the rover's autonomous navigation.

rebuild observation equation. The working principle of this solution is shown in Fig. 6:

When the rover is in motion, by fusing the matrix $\widetilde{C^{b}}$ with the matrix $\hat{C}^{b}$ the platform misalignment angle vector $\boldsymbol{\varphi}^{n}$ and the gyro drift vector $\boldsymbol{\varepsilon}^{b}$ can be estimated in real time. So the attitude divergence speed can be slowed

down largely by using the estimate of $\varepsilon^{b}$ to correct the gyro's output, and the high precision attitude can be determined by using the estimate of $\varphi^{n}$ to correct the

attitude matrix $\hat{\boldsymbol{C}}_{b}{ }^{n}$. According to the rover's movement characteristics, when it does not sideslip and skip on the surface of Mars, the transverse and vertical velocities in rover body coordinate are zero in theory. Therefore, velocity error $\delta \boldsymbol{V}^{n}$ can be estimated by taking the transverse and vertical velocities in rover body coordinate as measurement variables, and using the estimate of velocity error $\delta \hat{\boldsymbol{V}}^{n}$ to correct the velocity outputted by SINS can obtain a higher accuracy velocity. The precision of rover's position can be improved much more with using the integration of $\delta \hat{V}^{n}$ to correct the position determined by SINS.

\subsection{SINS/CNS autonomous navigation system model}

According to the attitude error model, gyro error model, velocity error equation and position error Eqs. (11)-(14), the system state equation can still be established as follows:

$$
(t)=\mathbf{F}(t) \boldsymbol{X}(t)+\boldsymbol{G}(t) \boldsymbol{W}(t)
$$

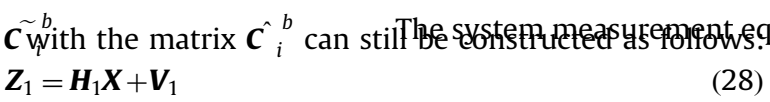

The following equation can be used to transform the velocity $\mathbf{v}^{\wedge}$ in the coordinate system $n$ to $\hat{\mathbf{v}}^{\hat{b}}$ in the coordinate system $b$ :

$\boldsymbol{v}^{\wedge}=\stackrel{\boldsymbol{C}}{b}^{\wedge}{ }^{n}$

From the Eq. (20), ther e is $\boldsymbol{C}^{b}{ }_{n}=\hat{\boldsymbol{C}}^{b}{ }_{n}\left(\boldsymbol{I}_{3} \times 3-\left[\varphi^{n} \times\right]\right)$ over, according to $\overline{\boldsymbol{v}}^{\wedge}{ }_{4} \delta \boldsymbol{v}{ }_{n}$ and ${ }_{x} \overline{\boldsymbol{v}} \theta, v_{z} \boldsymbol{C}={ }^{b} \boldsymbol{\theta}$, the difference between $\mathbf{v}^{\prime}$ and $\boldsymbol{b}^{b}$ can be expressed as follows:

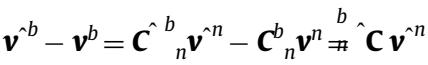

$$
\begin{aligned}
& -\hat{\boldsymbol{C}^{b}}{ }_{n}\left(\boldsymbol{I}_{3 \times 3}-\left[\boldsymbol{\varphi}^{n} \times\right]\right)\left(\boldsymbol{v}^{\wedge}-\delta \boldsymbol{v}^{n}\right)=\hat{\boldsymbol{C}}^{\hat{b}_{n}}\left[\boldsymbol{\varphi}^{n} \times\right] \boldsymbol{v}^{n}+\hat{\boldsymbol{C}}^{b}{ }_{n} \delta \boldsymbol{v}^{n}(30)
\end{aligned}
$$

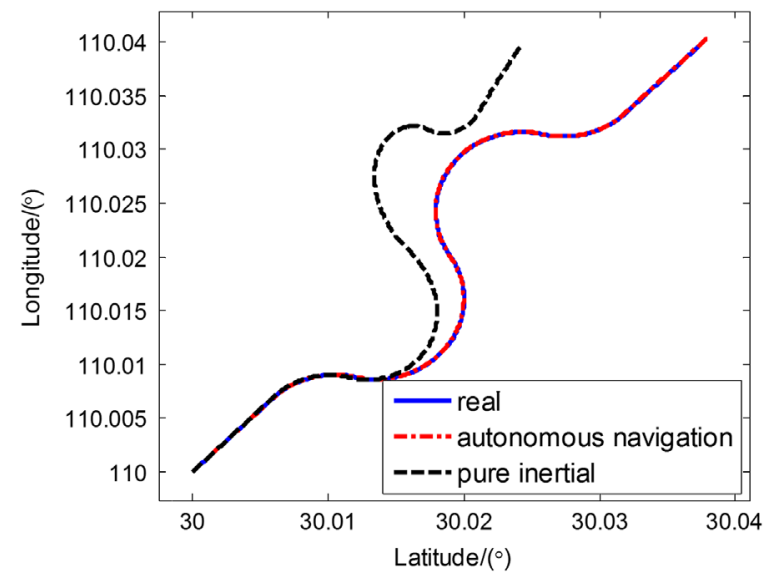

Fig. 7. The real track and the tracks calculated by autonomous navigation and pure inertial navigation.

Let $\boldsymbol{Z}_{2}=\left[\begin{array}{c}v_{{ }^{\prime}}^{b}-v_{x}^{b} \\ v^{\wedge}{ }^{b} v_{z}^{b}\end{array}\right]=\boldsymbol{H}_{2} \boldsymbol{X}+\boldsymbol{V}_{2}$, where: $\mathbf{H}_{2}$ is the velocity error measurement matrix, and $\boldsymbol{V}_{2}$ is the measurement noise. Let $\hat{\boldsymbol{C}}^{b}{ }_{n}=\left[\begin{array}{ccc}T_{11} & T_{12} & T_{13} \\ T_{21} & T_{22}\end{array}\right]$. Substituting it into Equ. (30), $\boldsymbol{H}_{2}$ can be shown as follows:

$$
\begin{aligned}
& \left.\boldsymbol{H}_{2}=\boldsymbol{H}_{21} \boldsymbol{H}_{22} \mathbf{0}_{2 \times 2} \mathbf{0}_{27)^{6}}^{\boldsymbol{X}} \quad\right] \\
& \text { where: }
\end{aligned}
$$

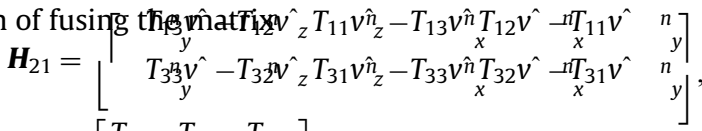

$$
\begin{aligned}
& \boldsymbol{H}_{22}=\left[\begin{array}{lll}
T_{11} & T_{12} & T_{13} \\
T_{31} & T_{32} & T_{33}
\end{array}\right]
\end{aligned}
$$

According to the measurement equations (28) and (31), the system measurement equation can be established as follows:

$$
v^{b}=\left[\begin{array}{l}
\boldsymbol{Z}_{1} \\
\boldsymbol{Z}_{2}
\end{array}\right]=\boldsymbol{H} \boldsymbol{X}+\boldsymbol{V}
$$

where, $\boldsymbol{H}=\left[\begin{array}{c}\boldsymbol{H}_{11} \mathbf{0}_{3 \times 3} \boldsymbol{H}_{13} \mathbf{0}_{3 \times 6} \boldsymbol{H}_{21} \\ \boldsymbol{H}_{22} \mathbf{0}_{2 \times 2} \mathbf{0}_{2 \times 6}\end{array}\right], \mathbf{V}$ is the measurement noise. 


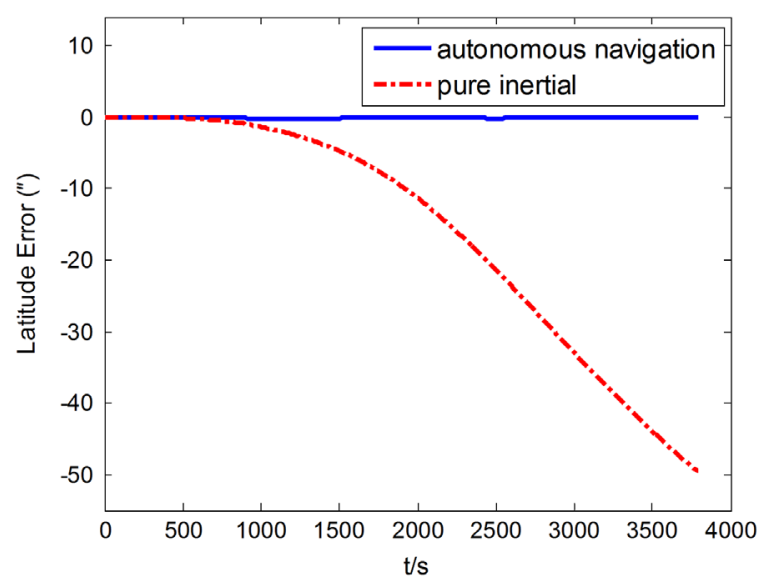

Fig. 8. The latitude error.

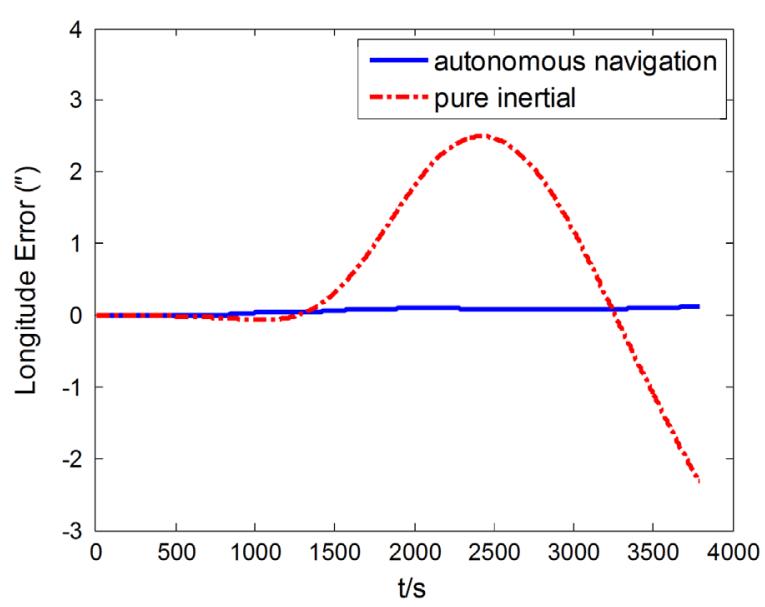

Fig. 9. The longitude error.

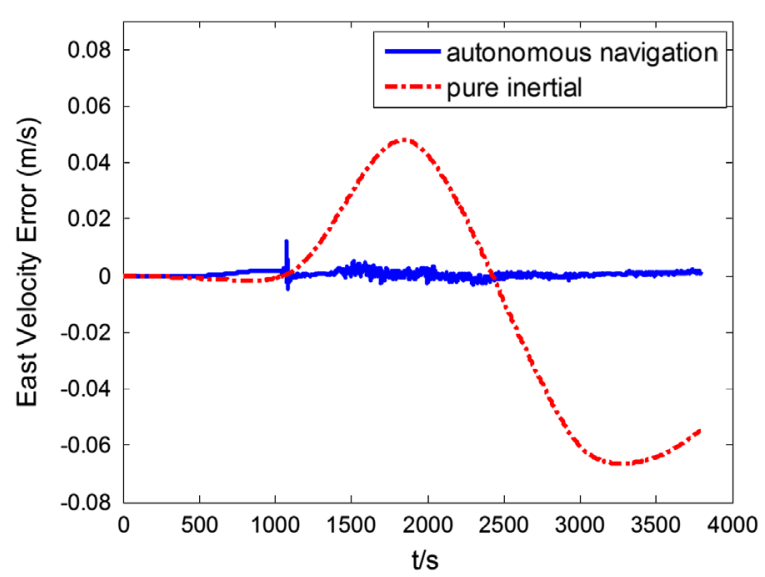

Fig. 10. The east velocity error.

\subsection{Simulation analysis}

The initial position and attitude of the track are defined as: latitude $30^{\circ} \mathrm{N}$, longitude $110^{\circ} \mathrm{E}$, with pitch angle $0^{\circ}$, roll angle $0^{\circ}$, yaw angle $60^{\circ}$. The IMU and star sensor are the same as shown in Section 2.3.5.

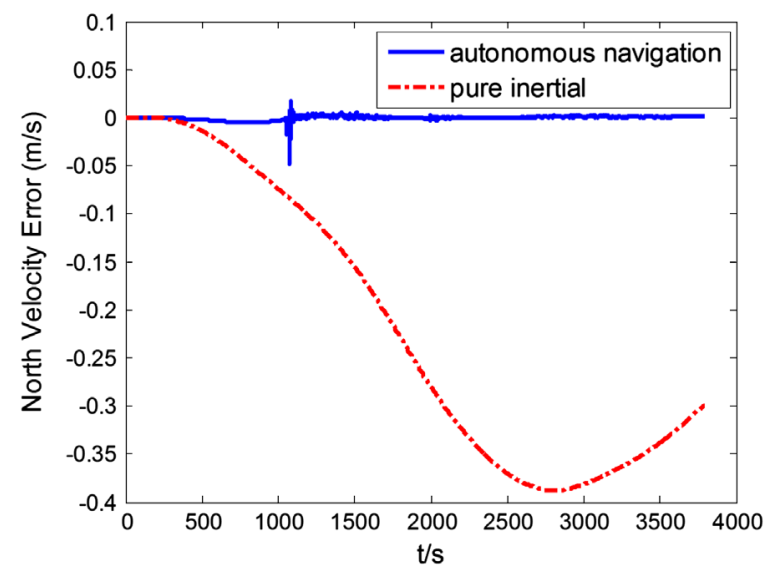

Fig. 11. The north velocity error.

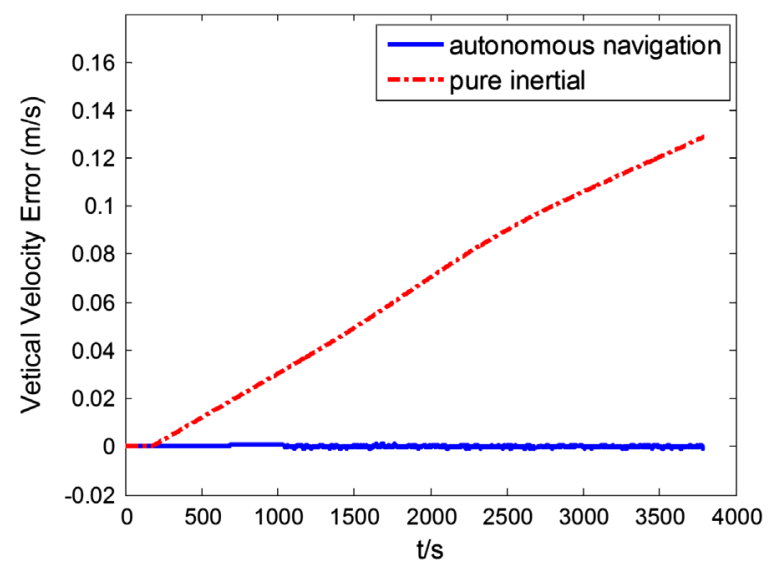

Fig. 12. The vertical velocity error.

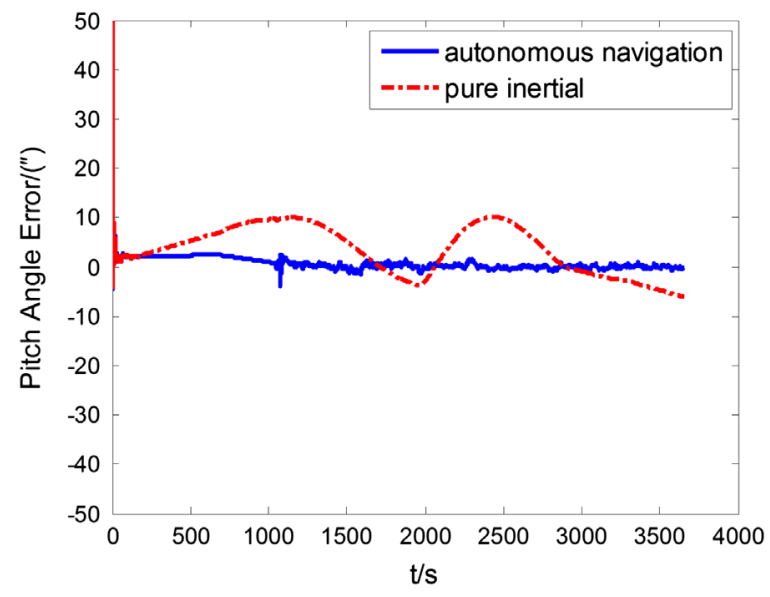

Fig. 13. The error of pitch angle.

At the end of SINS initial alignment, the proposed autonomous navigation method starts to calculate. Because the Kalman filtering period is $0.01 \mathrm{~s}$ and the data update rate of star sensor is $1 \mathrm{~Hz}$. Before the arrival of the data from star sensor, the transverse and vertical velocities in rover body coordinate system are used as one and only measurement. The matrix $\widetilde{\boldsymbol{C}}_{i}^{b}$ is fused with the matrix $\hat{\boldsymbol{C}}_{i}^{b}$ at 


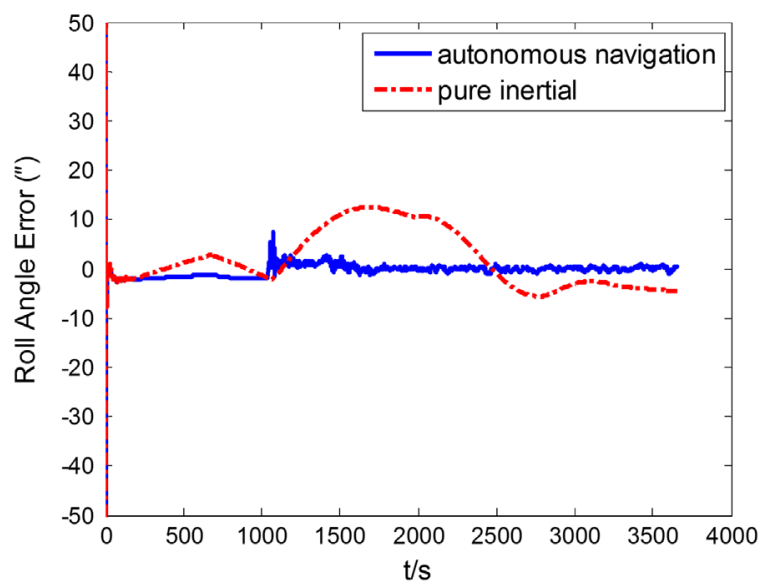

Fig. 14. The error of roll angle.

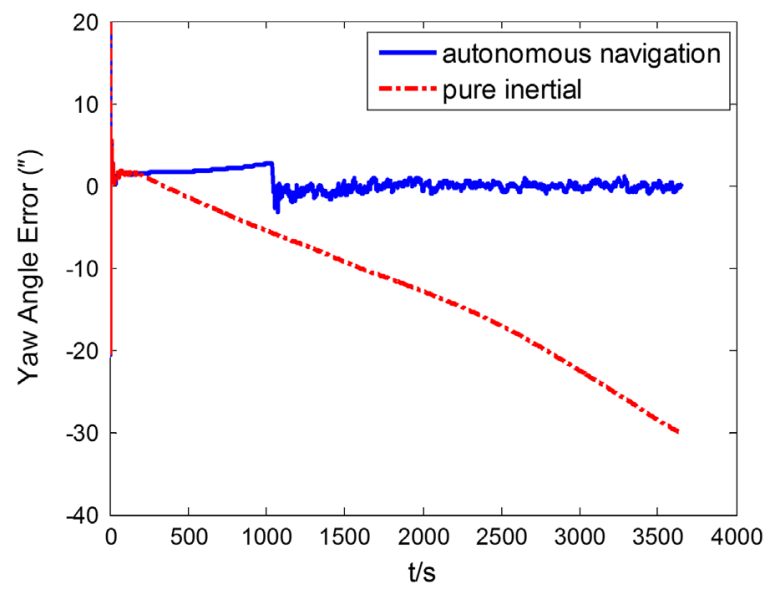

Fig. 15. The error of yaw angle.

the same time when the data from star sensor arrive. The simulation results are shown as follows:

Because the estimate of velocity error can be obtained exactly in this proposed solution, the velocity and position of rover can be corrected by the $\delta \hat{\boldsymbol{V}}^{n}$ and the integration of $\delta \hat{\boldsymbol{V}}^{n}$, respectively. Therefore, either the position error or the velocity error is very small when calculated by the autonomous navigation method, and the obtained track is coincident with the real track. The conclusions above are shown in Figs. 7-12. However, because the inertial instruments errors accumulate with time, the position error and velocity error all diverge with time in the pure inertial navigation method, and the calculated track is more and more deviated from the real track. In the presented autonomous navigation method for rover, we fuse the matrix $\hat{\boldsymbol{C}}_{i}^{b}$ with the matrix $\hat{\boldsymbol{C}^{\wedge}}{ }_{i}^{b}$, so the gyro drift vector $\boldsymbol{\varepsilon}^{b}$ and the platform misalignment angle vector $\varphi^{n}$ can be estimated in real time. Therefore, by using the estimates of $\boldsymbol{\varepsilon}^{b}$ and $\boldsymbol{\varphi}^{n}$ to correct the gyro's output and the attitude matrix $\hat{\boldsymbol{C}}_{b}^{n}$ respectively, the rover's attitude can be calcu-lated with high precision. This point can be supported by Figs. 13-15. The attitude errors are very small when calculated by the autonomous navigation method. But when the pure inertial navigation method is used to propagate the rover's attitude, the errors of pitch angle and roll angle oscillate with time, and the error of yaw angle deviates with time. This results from the influence of gyro drift and the rover's track.

Combing the simulation results of Figs. 7-15, it can be seen that the autonomous navigation method has shown superior navigation performance. Therefore, the presented method can meet the requirements of long time, high precision and reliability autonomous navigation for rovers.

\section{Conclusions}

In order to determine the position and attitude of a rover after it lands on the Mars. A novel initial alignment method including coarse and fine alignment in static mode is proposed. During coarse alignment, the pitch and roll angle of the rover is determined by using the measurement from its accelerometers. The position and yaw angle of the rover's is determined from the output of star sensor and time. Based on the results of coarse alignment, the fine alignment uses the zero velocity (static) observation and the output of star sensor to estimate the misalignment angles, latitude error and longitude error and inertial sensor errors. These estimates are subsequently used to correct the rover's initial attitude and position errors.

An autonomous navigation approach based on star sensor and SINS for rover is proposed. It employs Kalman filter to integrated star sensor and inertial sensor measurements to overcome the weakness of SINS. It takes the advantages of both inertial navigation and celestial navigation. Furthermore, the Kalman filter estimates and compensates the SINS errors in real time to prevent the accumulation of errors in navigation solution. This method has the potential to realize the high accuracy navigation for Mars rover in the future.

\section{Acknowledgments}

This work was supported by the National Natural Science Foundation of China (NSFC) under Grant no. 61233005, the State Key Lab Foundation of Astronautical Dynamics of China under Grant no. 2012ADL-DW0201 and the Aeronautical Science Foundation of China under Grant no. 20110151002. The authors also thank M.S. He Zhu and Zhang Qing for their valuable suggestions.

\section{References}

[1] Di Kaichang, A review of Spirit and Opportunity Rover Localization Methods, Spacecr. Eng. 18 (5) (2009) 1-5.

[2] A. Ellery, et al., A European robotic astrobiology-focussed Mars mission proposal (in press), Acta Astronaut. (2003).

[3] L. Yenilmez, H. Temeltas Autonomous navigation for

planetptgration by a mobile robot, 2003. RAST'03. in: Proceedings of IEEE International Conference on. Recent Advances in Space Technologies. 2003: 397-402.

[4] R. Li, K. Di, L.H. Matthies, et al., Rover localization and landing-site mapping technology for the 2003 Mars exploration rover mission, Photogramm. Eng. Remote Sens. 70 (1) (2004) 77-90.

[5] R.P. De Paula, C.D. Edwards Jr, E. Flamini, Evolution of the

commications systems and technology for Mars exploration, Acta Astro-naut. 51 (1) (2002) 207-212. 
[6] Z.h.u. Shengying, C.u.i. Pingyuan, C.u.i. Hutao, Shao Wei, Autonomous position and attitude determination for interplanetary landers based on landmark observation angles, Acta Aeronaut. ET Astronaut. Sin. 31 (2) (2010) 318-326.

[7] E. Gai, Guidance, navigation, and control from instrumentation to information management, J. Guid. Control and Dyn. 19 (1) (1996) $10-14$.

[8] Fuke Y. Krotkov E. Dead reckoning for a lunar rover on uneterirain, 1996 In: Proceedings of the 1996 IEEE International Con-ference on Robotics and Automation 1996, 1: 411-416.

[9] L.u.o. Qiangli, H.a.n. Junhai, Research on SINS and OD integrated navigation system for land vehicles, Navig. Control 10 (4) (2011) 6-11.

[10] S.B. Goldberg, M.W. Maimone, L. Matthies, Stereo vision and rovernavigation software for planetary exploration, in: Proceedings, of the 2002 IEEE Aerospace Conference 2002, 5: 5-2025-5-2036 5.

[11] J. Kim, S. Rock, Feedback dual controller design and its application to monocular vision-based docking, J. Guid., Control Dyn. 32 (4) (2009) $1134-1142$.

[12] M.L. Thein, D.A. Quinn, D.C. Folta, Celestial navigation (CelNav): lunar surface navigation, in: Proceedings of the 2008 AIAA/AAS Astrodynamics Specialist Congress and Exposition. 2008: 1-19.

[13] B.P. Malay, R. Fahey, Celestial navigation on the surface of Mars, Adv. Astronaut. Sci. 112 (2002) 487-511.

[14] D.A. Sigel, D. Wettergreenm, Star tracker celestial

localisyastom for a lunar rover, Intelligent Robots and Systems, 2007. IROS 2007. In: Proceedings of the IEEE/RSJ International Conference on IEEE, 2007: 2851-2856.

[15] J. Enright, T. Barfoot, M. Soto, Star tracking for planetary rovers, in: Proceedings of the 2012 IEEE Aerospace Conference, 2012: 1-13.

[16] X. Ning, J. Fang, A new autonomous celestial navigation method

for the lunar rover, Robot. Auton. Syst. 57 (1) (2009) 48-54.

(2011) 1631-1647.

[18] K.S. Ali, C.A. Vanelli, J.J. Biesiadecki, et al. Attitude and position estimation on the Mars exploration rovers, systems, man and cybernetics, in: Proceedings of the 2005 IEEE International Conference on IEEE, 2005, 1: 20-27.

[19] X. Wang, Q. Zhang, H. Li, An autonomous navigation scheme based on starlight, geomagnetic and gyros with information fusion for small satellites, Acta Astronaut. 94 (2) (2014) 708-717.

[20] W. Xiaojuan, W. Xinlong, A SINS/CNS deep integrated

navigatethod based on mathematical horizon reference, Aircr. Eng. Aerosp.Technol. 83 (1) (2011) 26-34.

[21] F. Xu, J. Fang, Velocity and position error compensation using strapdown inertial navigation system/celestial navigation system integration based on ensemble neural network, Aerosp. Sci. Technol. 12 (4) (2008) 302-307.

[22] P. Singla, J.L. Crassidis, J.L. Junkins, Spacecraft angular rate estimation algorithms for star tracker-based attitude determination, Adv. Astronaut. Sci. 114 (2003) 1303-1316.

[23] C.C. Liebe, Star trackers for attitude determination, Aerospace and Electronic Systems Magazine, IEEE 10 (6) (1995) 10-16.

[24] A. Trebi-Ollennu, T. Huntsberger, Y. Cheng, et al., Design and analysis of a sun sensor for planetary rover absolute heading detection, IEEE Trans. Robot. Autom. 17 (6) (2001) 939-947.

[25] X. Ning, L. Liu, A two-mode INS/CNS navigation method for lunar rovers, IEEE Trans. Instrum. Meas. (2014) 1-10.

[26] R.L. Farrenkopf, R.G. Reynolds, Analytics steady-state accuracy for two common spacecraft attitude estimators, J. Guid. Control 1 (4) (1978) 282-284.

[27] F.L. Markley, Attitude error representations for Kalman

filtering \&uid. Control Dyn. 26 (2) (2003) 311-317. 